\title{
AN ITERATIVE PROCESS OF GENERALIZED LIPSCHITIZIAN MAPPINGS IN UNIFORMLY CONVEX BANACH SPACES
}

\author{
A. RAZANI
}

Received 23 December, 2020

\begin{abstract}
In this article, three generalized Lipschitizian mappings in the uniformly convex Banach spaces are considered. By defining an iterative process, the existence of a common fixed point of this iteration is proved. An example is presented to guarantee the convergence of the iteration.
\end{abstract}

2010 Mathematics Subject Classification: 47H10; 54H10

Keywords: nonexpansive semigroups, iterative approximation, generalized Lipschitizian mappings

\section{INTRODUCTION}

In computational mathematics, an iterative method is a mathematical procedure that uses an initial value to generate a sequence of improving approximate solutions for a class of problems, in which the $n-t h$ approximation is derived from the previous ones. If an equation can be put into the form $f(x)=x$, and a solution $x$ is an attractive fixed point of the function $f$, then one may begin with a point $x_{1}$ in the basin of attraction of $x$, and let $x_{n+1}=f\left(x_{n}\right)$ for $n=1$, and the sequence $\left\{x_{n}\right\}_{n \geq 1}$ will converge to the solution $x$. Here $x_{n}$ is the $n$-th approximation or iteration of $x$ and $x_{n+1}$ is the next or $n+1$ iteration of $x$ (see [4,7,9-16], and reference therein).

Let $C$ be a nonempty subset of a Banach space $E, T: C \longrightarrow C$ be a mapping and

$$
F(T)=\{x \in C: T x=x\}
$$

denotes the set of fixed points of $T$. A mapping $T$ is said to be asymptotically nonexpansive, if there exists a sequence $\left\{k_{n}\right\}$ of positive numbers with $\lim _{n \longrightarrow \infty} k_{n}=1$ such that for $x, y \in C$ and $n \geq 1$,

$$
\left\|T^{n} x-T^{n} y\right\| \leq k_{n}\|x-y\| .
$$

The study of iterative construction for fixed points of asymptotically nonexpansive mappings began in 1975. Baillon [3] proved, if $C$ is a nonempty, closed and convex subset of a Hilbert space $\mathcal{H}$ and $T: C \rightarrow C$ is a nonexpansive mapping such that 
$F(T) \neq \varnothing$, then for every $x \in C$, the Cesáro means

$$
T_{n} x=\frac{1}{n+1} \sum_{j=0}^{n} T^{j} x
$$

is weakly convergent to a fixed point of $T$. Then, Shimizu et al. [18] studied the convergence of the following approximated sequence for an asymptotically nonexpansive mapping in Hilbert space,

$$
\begin{aligned}
x_{0} & =x \in C, \\
x_{n+1} & =\alpha_{n} x+\left(1-\alpha_{n}\right) \frac{1}{n+1} \sum_{j=0}^{n} T^{j} x_{n},
\end{aligned}
$$

where $\left\{\alpha_{n}\right\}$ is a real sequence satisfying $0<\alpha_{n} \leq 1$ and $\alpha_{n} \longrightarrow 0$ as $n \longrightarrow \infty$. They proved that $\left\{x_{n}\right\}$ is strongly convergent to an element of $F(T)$. We recall the definition of uniformly convex space (see [2,6] for more details).

Definition 1. A Banach space $E$ is said to be strictly convex if

$$
\|x+y\|<2
$$

for all $x, y \in E$ with $\|x\|=\|y\|=1$ and $x \neq y$. We recall that a Banach space $E$ is called uniformly convex, if for each $\varepsilon>0$ there is a $\delta>0$ such that if $\|x\|=\|y\|=1$ then

$$
\left\|\frac{(x+y)}{2}\right\| \leq 1-\delta
$$

It is obvious that uniform convexity implies strict convexity.

In 1991, [23] proved the characterization of uniform convexity as follows.

Theorem 1. A Banach space $E$ is uniformly convex if and only if for each fixed number $r>0$, there exists a continuous function $\varphi:[0, \infty) \rightarrow[0, \infty), \varphi(s)=0 \Longleftrightarrow$ $s=0$, such that

$$
\|\lambda x+(1-\lambda) y\|^{2} \leq \lambda\|x\|^{2}+(1-\lambda)\|y\|^{2}-\lambda(1-\lambda) \varphi(\|x-y\|)
$$

for all $\lambda \in[0,1]$ and all $x, y \in E$ such that $\|x\| \leq r$ and $\|y\| \leq r$.

Shioji et al. [19] studied the strongly convergence of the sequence

$$
\begin{aligned}
x_{0} & =x \in C, \\
x_{n+1} & =\alpha_{n} x+\left(1-\alpha_{n}\right) \frac{1}{n+1} \sum_{j=0}^{n} T^{j} x_{n},
\end{aligned}
$$

in uniformly convex Banach spaces with uniformly Gâteaux differentiable norms.

Also, for an asymptotically nonexpansive mapping $T$, Tan et. al [22] defined the modified Ishikawa iterations process

$$
x_{n+1}=t_{n} T^{n}\left(s_{n} T^{n} x_{n}+\left(1-s_{n}\right) x_{n}\right)+\left(1-t_{n}\right) x_{n},
$$


where $\left\{t_{n}\right\}$ and $\left\{s_{n}\right\}$ are real sequences such that $\left\{t_{n}\right\}$ is bounded away from 0 and 1 and $\left\{s_{n}\right\}$ bounded away from 1 . They proved the sequence $\left\{x_{n}\right\}$ is weakly convergent to a fixed point of $T$.

Definition 2. Let $E$ be a Banach space. $E$ is said to satisfy Opial's condition if for each sequence $\left\{x_{n}\right\}$ in $E$ the condition $x_{n} \rightarrow x$ implies

$$
\limsup _{n \longrightarrow \infty}\left\|x_{n}-x\right\|<\limsup _{n \longrightarrow \infty}\left\|x_{n}-y\right\|
$$

for all $y \in E$ and $y \neq x$.

Definition 3. Let $E$ be an arbitrary real Banach space with norm $\|$.$\| and E^{*}$ be the dual space of $E$. The duality mapping $J: E \longrightarrow E^{*}$ is defined by

$$
J x=\left\{f \in E^{*}:\langle x, f\rangle=\|x\|^{2},\|f\|=\|x\|\right\},
$$

where $\langle x, f\rangle$ denotes the value of the continuous linear function $f \in E^{*}$ at $x \in E$.

Lemma 1 ([21]). Let $\left\{\delta_{n}\right\},\left\{\beta_{n}\right\}$ and $\left\{\gamma_{n}\right\}$ be three sequences of nonnegative numbers satisfying the recursive inequality

$$
\delta_{n+1} \leq \beta_{n} \delta_{n}+\gamma_{n} \text { for all } n \geq 1,
$$

if $\beta_{n} \geq 1, \sum_{n=1}^{\infty}\left(\beta_{n}-1\right)<\infty$ and $\sum_{n=1}^{\infty} \gamma_{n}<\infty$, then $\lim _{n \rightarrow \infty} \delta_{n}$ exists.

Lemma 2 ([17]). Let $E$ be a uniformly convex Banach space. Assume $\left\{t_{n}\right\}$ is a sequence of real numbers in $(0,1)$ bounded away from 0 and 1 . If $\left\{x_{n}\right\}$ and $\left\{y_{n}\right\}$ are two sequences of $E$ such that for some $a \geq 0$

$$
\limsup _{n \longrightarrow \infty}\left\|x_{n}\right\| \leq a, \limsup _{n \longrightarrow \infty}\left\|y_{n}\right\| \leq a \text { and } \limsup _{n \longrightarrow \infty}\left\|t_{n} x_{n}+\left(1-t_{n}\right) y_{n}\right\|=a,
$$

then $\lim _{n \rightarrow \infty}\left\|x_{n}-y_{n}\right\|=0$.

Lemma 3 ([1]). Let $\left\{x_{n}\right\}$ be a bounded sequence in a uniformly convex Banach space $E$. If $w_{w}\left(\left\{x_{n}\right\}\right)=\{x\}$, then $x_{n} \rightarrow x$, where $w_{w}\left(\left\{x_{n}\right\}\right)=\left\{x \in E: \exists x_{n j} \rightarrow x\right\}$ denotes the weak $w$-limit set of $\left\{x_{n}\right\}$.

In 2001, Jung et al. [8] introduced the following class of mappings.

Definition 4. A mapping $T: C \rightarrow C$, where $C$ is a nonempty subset of Banach space $E$, is said to be a generalized Lipschitzian mapping if

$\left\|T^{n} x-T^{n} y\right\| \leq a_{n}\|x-y\|+b_{n}\left(\left\|x-T^{n} x\right\|+\left\|T^{n} y-y\right\|\right)+c_{n}\left(\left\|x-T^{n} y\right\|+\left\|T^{n} x-y\right\|\right)$

for each $x, y \in C$ and $n \geq 1$, where $a_{n}, b_{n}$ and $c_{n}$ are nonnegative constants such that there exists an integer $n_{0}$ such that $b_{n}+c_{n}<1$, for all $n>n_{0}$. 
Definition 5. A mapping $T: C \rightarrow C$, where $C$ is a nonempty subset of Banach space $E$, is said to be a uniformly generalized Lipschitzian mapping if

$$
\left\|T^{n} x-T^{n} y\right\| \leq a\|x-y\|+b\left(\left\|x-T^{n} x\right\|+\left\|T^{n} y-y\right\|\right)+c\left(\left\|x-T^{n} y\right\|+\left\|T^{n} x-y\right\|\right)
$$

for each $x, y \in C$ where $a, b$ and $c$ are nonnegative constants and $b+c<1$.

Clearly, every Lipschitzian mapping is a generalized Lipschitzian mapping. But the vice versa is not necessarily true. See the following example.

Example 1. Let $E=\mathbb{R}$ be the set of real numbers and $C=[0, \infty)$. For each $x \in C$, we define

$$
T x=\left\{\begin{array}{lll}
\frac{r x}{1+x} & \text { if } & x \in\left[0, \frac{1}{4}\right], \\
0 & \text { if } & x \in\left(\frac{1}{4}, \infty\right),
\end{array}\right.
$$

where $0<r<\frac{1}{4}$. Then $T: C \longrightarrow C$ is not continuous at $x=\frac{1}{4}$ and hence $T$ is not a Lipschitzian mapping. Set $C_{1}=\left[0, \frac{1}{4}\right]$ and $C_{2}=\left(\frac{1}{4}, \infty\right)$. In order to prove $T: C \longrightarrow C$ is a generalized Lipschitzian mapping, we need the following steps: for all $x, y \in C_{1}$ and $n \geq 1$,

$$
|T x-T y|=\left|\frac{r x}{1+x}-\frac{r y}{1+y}\right|=\left|\frac{r x(1+y)-r y(1+x)}{(1+x)(1+y)}\right| \leq r|x-y|
$$

and

$$
\left|T^{2} x-T^{2} y\right|=\left|\frac{r T x}{1+T x}-\frac{r T y}{1+T y}\right| \leq r|T x-T y| \leq r^{2}|x-y| .
$$

By induction, for all $n \geq 1$

$$
\left|T^{n} x-T^{n} y\right| \leq r^{n}|x-y|+r^{n}\left(\left|x-T^{n} x\right|+\left|y-T^{n} y\right|\right)+r^{n}\left(\left|x-T^{n} y\right|+\left|y-T^{n} x\right|\right) .
$$

For all $x, y \in C_{2}$ and $n \geq 1$,

$$
\left|T^{n} x-T^{n} y\right|=0 \leq|x-y| .
$$

For $x \in C_{1}$ and $y \in C_{2}$,

By induction, for $n \in \mathbb{N}$,

$$
|T x-T y|=\left|\frac{r x}{1+x}-0\right| .
$$

$$
T^{n+1} x=\frac{r T^{n} x}{1+T^{n} x} \leq r T^{n} x \leq r^{n+1} x,
$$

and by a computation

$$
\begin{aligned}
\left|T^{n} x-T^{n} y\right| & =\left|T^{n} x-0\right| \\
& \leq r^{n}\left|(x-y)+\left(y-T^{n} x\right)+\left(T^{n} x-x\right)+x\right| \\
& \leq r^{n}\left(|x-y|+\left|T^{n} x-x\right|+\left|y-T^{n} x\right|+|x+y-0|\right) \\
& \leq r^{n}|x-y|+r^{n}\left(\left|x-T^{n} x\right|+\left|y-T^{n} y\right|\right)+r^{n}\left(\left|x-T^{n} y\right|+\left|y-T^{n} x\right|\right) .
\end{aligned}
$$


Thus inequalities (1.4), (1.5) and (1.6) imply that $T: C \longrightarrow C$ is a generalized Lipschitzian mapping.

Lemma 4. Let $C$ be a bounded, closed and convex subset of a uniformly convex Banach space $E$ and $T$ be a uniformly generalized Lipschitzian mapping of $C$ into itself. Then, for any $\varepsilon>0$, there exists a positive number $\xi(\varepsilon)$ such that $\|T x-x\|<\varepsilon$ for all $x \in\left[x_{0}, x_{1}\right]$ whenever for $x_{0}, x_{1} \in C,\left\|T x_{0}-x_{0}\right\| \leq \xi(\varepsilon)$ and $\left\|T x_{1}-x_{1}\right\| \leq \xi(\varepsilon)$, where

$$
\left[x_{0}, x_{1}\right]=\left\{\lambda x_{1}+(1-\lambda) x_{0}: 0 \leq \lambda \leq 1\right\} .
$$

Proof. Fix $\varepsilon>0$ and $x \in\left[x_{0}, x_{1}\right]$. Then $x=\lambda x_{1}+(1-\lambda) x_{0}$ for some $\lambda$ with $0 \leq \lambda \leq 1$. We consider two cases, $\left\|x_{1}-x_{0}\right\|<\varepsilon_{1}$ and $\left\|x_{1}-x_{0}\right\| \geq \varepsilon_{1}$.

If $\left\|x_{1}-x_{0}\right\|<\varepsilon_{1}$ then it is obvious that $\left\|x-x_{0}\right\|<\varepsilon_{1}$, where $\varepsilon_{1}=\frac{1-(b+c)}{1+a+2 c} \frac{\varepsilon}{2}$. Since

$$
\begin{aligned}
\|T x-x\| \leq & \left\|T x-T x_{0}\right\|+\left\|T x_{0}-x_{0}\right\|+\left\|x_{0}-x\right\| \\
\leq & a\left\|x_{0}-x\right\|+b\left(\|T x-x\|+\left\|T x_{0}-x_{0}\right\|\right) \\
& +c\left(\left\|x_{0}-T x\right\|+\left\|T x_{0}-x\right\|\right)+\left\|T x_{0}-x_{0}\right\|+\left\|x_{0}-x\right\| \\
\leq & a\left\|x_{0}-x\right\|+b\left(\|T x-x\|+\left\|T x_{0}-x_{0}\right\|\right) \\
& +c\left(\|x-T x\|+\left\|T x_{0}-x_{0}\right\|+2\left\|x_{0}-x\right\|\right)+\left\|T x_{0}-x_{0}\right\|+\left\|x_{0}-x\right\|
\end{aligned}
$$

and so

$$
\|T x-x\| \leq \frac{1+a+2 c}{1-(b+c)}\left\|x_{0}-x\right\|+\frac{b+c+1}{1-(b+c)}\left\|x_{0}-T x_{0}\right\| .
$$

Thus if $\left\|T x_{0}-x_{0}\right\| \leq \xi(\varepsilon)<\frac{1-(b+c)}{1+b+c} \frac{\varepsilon}{2}$, then $\|T x-x\|<\varepsilon$.

If $\left\|x_{0}-x_{1}\right\| \geq \varepsilon_{1}$, let $d_{0}$ denote the diameter of $C$. For any nonnegative number $0 \leq \lambda \leq 1$, we consider three cases:

Case 1) If $0 \leq \lambda<\frac{\varepsilon_{1}}{d_{0}}$, then

$$
\left\|x_{0}-x\right\|=\lambda\left\|x_{1}-x_{0}\right\|<\frac{\varepsilon_{1}}{d_{0}}\left\|x_{1}-x_{0}\right\|=\varepsilon_{1} \frac{\left\|x_{1}-x_{0}\right\|}{d_{0}}<\varepsilon_{1} .
$$

Thus similar to above, if $\xi(\varepsilon)<\frac{1-(b+c)}{1+b+c} \frac{\varepsilon}{2}$, then inequation (1.7) implies $\|T x-x\|<\varepsilon$.

Case 2) If $1-\frac{\varepsilon_{1}}{d_{0}}<\lambda \leq 1$ or $0 \leq(1-\lambda)<\frac{\varepsilon_{1}}{d_{0}}$, so

$$
\left\|x_{1}-x\right\|=(1-\lambda)\left\|x_{1}-x_{0}\right\|<\varepsilon_{1},
$$

and similar to above we have $\|T x-x\|<\varepsilon$.

Case 3) If $\frac{\varepsilon_{1}}{d_{0}} \leq \lambda \leq 1-\frac{\varepsilon_{1}}{d_{0}}, y=T x$ implies

$$
\left\|y-x_{0}\right\|=\left\|T x-x_{0}\right\| \leq\left\|T x-T x_{0}\right\|+\left\|T x_{0}-x_{0}\right\| .
$$

By inequalities (1.2) and (1.7),

$$
\left\|y-x_{0}\right\| \leq \frac{a+b+c}{1-(b+c)}\left\|x-x_{0}\right\|+\frac{1+b+c}{1-(b+c)}\left\|T x_{0}-x_{0}\right\| \leq r \lambda\left\|x_{1}-x_{0}\right\|+h \xi(\varepsilon),
$$


where $r=\frac{a+b+c}{1-(b+c)}$ and $h=\frac{1+b+c}{1-(b+c)}$, and also

$$
\begin{aligned}
\left\|y-x_{1}\right\| & \leq \frac{a+b+c}{1-(b+c)}\left\|x-x_{1}\right\|+\frac{1+b+c}{1-(b+c)}\left\|T x_{1}-x_{1}\right\| \\
& \leq r(1-\lambda)\left\|x_{1}-x_{0}\right\|+h \xi(\varepsilon) .
\end{aligned}
$$

Set

Then

$$
z_{0}=\frac{y-x_{0}}{\lambda\left\|x_{1}-x_{0}\right\|} \text { and } z_{1}=\frac{x_{1}-y}{(1-\lambda)\left\|x_{1}-x_{0}\right\|} .
$$

and similarly

$$
\left\|z_{0}\right\| \leq \frac{r \lambda\left\|x_{1}-x_{0}\right\|+h \xi(\varepsilon)}{\lambda\left\|x_{1}-x_{0}\right\|} \leq r+\frac{h \xi(\varepsilon) d_{0}}{\varepsilon_{1}^{2}},
$$

On the other hand, for $\lambda$ with $\frac{\varepsilon_{1}}{d_{0}} \leq \lambda \leq 1-\frac{\varepsilon_{1}}{d_{0}}$,

$$
\left\|z_{1}\right\| \leq r+\frac{h \xi(\varepsilon) d_{0}}{\varepsilon_{1}^{2}} .
$$

$$
\left\|\lambda z_{0}+(1-\lambda) z_{1}\right\|=\frac{\left\|x_{1}-x_{0}\right\|}{\left\|x_{1}-x_{0}\right\|}=1 .
$$

Therefore using uniform convexity of $E$, we can choose $\xi(\varepsilon)$ so small that $\left\|z_{1}-z_{0}\right\|<\frac{\varepsilon}{d_{0}}$. Thus by $x=\lambda x_{1}+(1-\lambda) x_{0}$ and (1.10) we have

$$
\begin{aligned}
\|y-x\| & =\left\|(1-\lambda)\left(y-x_{0}\right)-\lambda\left(x_{1}-y\right)\right\| \\
& =\lambda(1-\lambda)\left\|x_{1}-x_{0}\right\|\left\|z_{1}-z_{0}\right\| \\
& <\varepsilon .
\end{aligned}
$$

Notice that $y=T x$, and this means $\|T x-x\|<\varepsilon$.

Lemma 5 ([20]). Let $C$ be a bounded, closed and convex subset of a uniformly convex Banach space $E$ and $T$ be a uniformly generalized Lipschitzian mapping of $C$ into itself. If $\left\{x_{j}\right\}$ is a sequence of $C$ such that $x_{j} \rightarrow x_{0}$ and $x_{j}-T x_{j} \longrightarrow 0$, then $x_{0}$ is a fixed point of $T$.

Proof. Since $\left\|(I-T) x_{j}\right\|=\varepsilon_{j} \longrightarrow 0$, for each $j$ we assume $\varepsilon_{j} \leq \xi\left(\varepsilon_{j-1}\right)<\varepsilon_{j-1}$, where $\xi(\varepsilon)$ for any $\varepsilon>0$ is the constant described in the conclusion of Lemma 4. Hence if $x \in \overline{C o}\left\{x_{j}: j \geq k\right\}$ then $\|x-T x\| \leq \varepsilon_{k-1}$. Since $\overline{C o}\left\{x_{j}: j \geq k\right\}$ is weakly compact, $x_{0} \in \overline{C o}\left\{x_{j}: j \geq k\right\}, k=1,2,3, \cdots$ and hence $\left\|x_{0}-T x_{0}\right\| \leq \varepsilon_{j}$, $j=1,2,3, \cdots$. This implies $\left\|T x_{0}-x_{0}\right\|=0$.

Let $C$ be a bounded, closed and convex subset of a Banach space $E$ and $\left\{T_{n}\right\}$ be a sequence of uniformly generalized Lipschitizian self-mappings of $C$, such that the set $F$ of common fixed points of $\left\{T_{n}\right\}$ is nonempty. Let $k_{n}=\frac{a_{n}+b_{n}+c_{n}}{1-\left(b_{n}+c_{n}\right)}$ for $T_{n}$ and $k_{n} \geq 1$ for all $n \geq 1$. For a given $x_{1} \in C$, we define the sequence $\left\{x_{n}\right\}$ by $x_{n+1}=T_{n} x_{n}$ for $n \geq 1$. 
Lemma 6. Let $E$ be a normed linear space and $C$ be a nonempty closed and convex subset of $E$. Let $T: C \longrightarrow C$ be a uniformly generalized Lipschitzian mapping. Let $\left\{x_{n}\right\}$ be defined by $x_{n+1}=T_{n} x_{n}$. If $\lim _{n \longrightarrow \infty}\left\|x_{n}-T^{n} x_{n}\right\|=0$ and $\lim _{n \longrightarrow \infty}\left\|x_{n}-x_{n+1}\right\|=0$, then $\lim _{n \longrightarrow \infty}\left\|x_{n}-T x_{n}\right\|=0$.

Proof. Let $r_{n}=\left\|T^{n} x_{n}-x_{n}\right\|$. Since $T$ is a uniformly generalized Lipschitzian mapping,

$$
\begin{aligned}
\left\|x_{n+1}-T x_{n+1}\right\| \leq & \left\|x_{n+1}-T^{n+1} x_{n+1}\right\|+\left\|T x_{n+1}-T^{n+1} x_{n+1}\right\| \\
\leq & r_{n+1}+a\left\|x_{n+1}-T^{n} x_{n+1}\right\|+b\left(\left\|x_{n+1}-T x_{n+1}\right\|\right. \\
& \left.+\left\|T^{n} x_{n+1}-T^{n+1} x_{n+1}\right\|\right)+c\left(\left\|x_{n+1}-T^{n+1} x_{n+1}\right\|\right. \\
& \left.+\left\|T^{n} x_{n+1}-T x_{n+1}\right\|\right) \\
\leq & r_{n+1}+a\left\|x_{n+1}-T^{n} x_{n+1}\right\|+b\left(\left\|T^{n} x_{n+1}-x_{n+1}\right\|\right. \\
& \left.+\left\|x_{n+1}-T x_{n+1}\right\|+\left\|T^{n+1} x_{n+1}-x_{n+1}\right\|\right) \\
& +c\left(\left\|x_{n+1}-T^{n+1} x_{n+1}\right\|+\left\|T^{n} x_{n+1}-x_{n+1}\right\|+\left\|x_{n+1}-T x_{n+1}\right\|\right),
\end{aligned}
$$

and

$$
\left\|x_{n+1}-T x_{n+1}\right\| \leq \frac{1+b+c}{1-(b+c)} r_{n+1}+\frac{a+b+c}{1-(b+c)}\left\|x_{n+1}-T^{n} x_{n+1}\right\| .
$$

Now we obtain $\left\|x_{n+1}-T^{n} x_{n+1}\right\|$ as

$$
\begin{aligned}
\left\|x_{n+1}-T^{n} x_{n+1}\right\| \leq & \left\|x_{n}-T^{n} x_{n}\right\|+\left\|x_{n+1}-x_{n}\right\|+\left\|T^{n} x_{n+1}-T^{n} x_{n}\right\| \\
\leq & r_{n}+\left\|x_{n+1}-x_{n}\right\|+a\left\|x_{n+1}-x_{n}\right\|+b\left(\left\|T^{n} x_{n}-x_{n}\right\|\right. \\
& \left.+\left\|T^{n} x_{n+1}-x_{n+1}\right\|\right)+c\left(\left\|x_{n+1}-T^{n} x_{n}\right\|+\left\|T^{n} x_{n+1}-x_{n}\right\|\right) .
\end{aligned}
$$

So

$$
\left\|T^{n} x_{n+1}-x_{n+1}\right\| \leq \frac{1+b+c}{1-(b+c)} r_{n}+\frac{1+a+2 c}{1-(b+c)}\left\|x_{n+1}-x_{n}\right\| .
$$

By relations (1.12) and (1.13),

$$
\begin{aligned}
\left\|x_{n+1}-T x_{n+1}\right\| \leq & \frac{1+b+c}{1-(b+c)} r_{n+1}+\frac{a+b+c}{1-(b+c)}\left\|T^{n} x_{n+1}-x_{n+1}\right\| \\
\leq & \frac{1+b+c}{1-(b+c)} r_{n+1}+\frac{a+b+c}{1-(b+c)}\left(\frac{1+b+c}{1-(b+c)} r_{n}\right. \\
& \left.+\frac{1+a+2 c}{1-(b+c)}\left\|x_{n+1}-x_{n}\right\|\right) .
\end{aligned}
$$

This completes the proof.

In the next section, based on [19] and [22], a modified version of the iterative process for three generalized Lipschitizian mappings is presented. Then the existence of a common fixed point for these three maps is proved. 


\section{AN ITERATIVE APPROXIMATION OF THREE GENERALIZED LIPSCHITIZIAN MAPPINGS}

In this section, we prove, if $T_{i}: C \longrightarrow C, i=1,2,3$, are three generalized Lipschitzian mappings, then the sequence $\left\{x_{n}\right\}$ which is defined by equation (2.1) converges to $q \in \bigcap_{i=1}^{3} F\left(T_{i}\right)$ in the uniformly convex Banach space.

The result presented in this section generalizes and improves the corresponding results, in [5,19] and [22].

Let $C$ be a nonempty subset of real Banach space $E$ and $T_{i}: C \longrightarrow C, i=1,2,3$, be three generalized Lipschitizian mappings. Consider the following iterative sequence $\left\{x_{n}\right\}$ which is defined by

$$
\begin{cases}x_{1} \in C, & \\ u_{n}=\frac{1}{n+1} \sum_{j=0}^{n} T_{1}^{j} x_{n}, & \\ z_{n}=\left(1-\lambda_{n}\right) x_{n}+\lambda_{n} u_{n} & n \geq 1, \\ y_{n}=\left(1-\beta_{n}\right) x_{n}+\beta_{n} T_{2}^{n} z_{n} & n \geq 1, \\ x_{n+1}=\left(1-\alpha_{n}\right) x_{n}+\alpha_{n} T_{3}^{n} y_{n} & n \geq 1,\end{cases}
$$

where $0<\alpha_{n}, \beta_{n}, \lambda_{n}<1$. We prove, if $T_{i}: C \longrightarrow C, i=1,2,3$, are three generalized Lipschitzian mappings, then the sequence $\left\{x_{n}\right\}$ which is defined by equation (2.1) converges to $q \in \bigcap_{i=1}^{3} F\left(T_{i}\right)$, in a uniformly convex Banach space.

Theorem 2. Let $E$ be a uniformly convex Banach space, satisfying Opial's condition and $C$ be a nonempty, closed and convex subset of $E . T_{i}: C \longrightarrow C$ are three generalized Lipschitizian mappings for $i=1,2,3$ satisfying

$\left\|T_{i}^{n} x-T_{i}^{n} y\right\| \leq a_{n}^{i}\|x-y\|+b_{n}^{i}\left(\left\|x-T_{i}^{n} x\right\|+\left\|T_{i}^{n} y-y\right\|\right)+c_{n}^{i}\left(\left\|x-T_{i}^{n} y\right\|+\left\|T_{i}^{n} x-y\right\|\right)$

for all $x, y \in C$ and $n \geq 1$, where $k_{n}^{i}=\frac{a_{n}^{i}+b_{n}^{i}+c_{n}^{i}}{1-\left(b_{n}^{i}+c_{n}^{i}\right)}$ and $\left\{k_{n}^{i}\right\} \subset[1, \infty)$ for $i=1,2,3$ satisfy $k_{n}^{i} \longrightarrow 1$ as $n \longrightarrow \infty$. Also $\sum_{n=1}^{\infty}\left(\eta_{n} k_{n}^{2} k_{n}^{3}-1\right)<\infty$ where $\eta_{n}=\max \left\{k_{j}^{1}, 1 \leq j \leq n\right\}$.

Let $\left\{x_{n}\right\}$ be the sequence defined by (2.1). If the following statements hold

(I) $F=\bigcap_{i=1}^{3} F\left(T_{i}\right) \neq \phi$

(II) $0<\liminf _{n \longrightarrow \infty} \alpha_{n} \leq \limsup _{n \rightarrow \infty} \alpha_{n}<1$

(III) $0<\liminf _{n \longrightarrow \infty} \beta_{n} \leq \limsup \sup _{n \rightarrow \infty} \beta_{n}<1$

(IV) $0<\liminf _{n \longrightarrow \infty} \lambda_{n} \leq \limsup \sup _{n \rightarrow \infty} \lambda_{n}<1$

then

(1) $\lim _{n \rightarrow \infty}\left\|x_{n}-q\right\|$ exists, for all $q \in F$.

(2) $\lim _{n \longrightarrow \infty}\left\|x_{n}-u_{n}\right\|=0$ and $\lim _{n \rightarrow \infty}\left\|x_{n}-T_{i} x_{n}\right\|=0(i=2,3)$.

(3) The sequence $\left\{x_{n}\right\}$ is weakly convergent to a common fixed point of $T_{i}(i=1,2,3)$. 
Proof. For any $q \in F$ and $i=1,2,3$, we get

$$
\begin{aligned}
\left\|T_{i}^{n} x_{n}-q\right\| & \leq a_{n}^{i}\left\|x_{n}-q\right\|+b_{n}^{i}\left\|T_{i}^{n} x_{n}-x_{n}\right\|+c_{n}^{i}\left(\left\|T_{i}^{n} x_{n}-q\right\|+\left\|x_{n}-q\right\|\right) \\
& \leq a_{n}^{i}\left\|x_{n}-q\right\|+b_{n}^{i}\left(\left\|T_{i}^{n} x_{n}-q\right\|+\left\|x_{n}-q\right\|\right)+c_{n}^{i}\left(\left\|T_{i}^{n} x_{n}-q\right\|+\left\|x_{n}-q\right\|\right),
\end{aligned}
$$

and so

$$
\left\|T_{i}^{n} x_{n}-q\right\| \leq \frac{a_{n}^{i}+b_{n}^{i}+c_{n}^{i}}{1-\left(b_{n}^{i}+c_{n}^{i}\right)}\left\|x_{n}-q\right\|=k_{n}^{i}\left\|x_{n}-q\right\| .
$$

In order to find a bound for $\left\|x_{n+1}-q\right\|$, we need to compute $\left\|u_{n}-q\right\|,\left\|z_{n}-q\right\|$ and $\left\|y_{n}-q\right\|$ as follows:

(i) Suppose $1 \leq j \leq n$, by inequality (2.2)

$$
\left\|u_{n}-q\right\| \leq \frac{1}{n+1}\left(\left\|x_{n}-q\right\|+\sum_{j=1}^{n} k_{j}^{1}\left\|x_{n}-q\right\|\right) \leq \eta_{n}\left\|x_{n}-q\right\| .
$$

(ii) The definition of $z_{n}$ in (2.1) implies

$$
\begin{aligned}
\left\|z_{n}-q\right\| & =\left\|\left(1-\lambda_{n}\right) x_{n}+\lambda_{n} u_{n}-q\right\| \\
& \leq\left(1-\lambda_{n}\right)\left\|x_{n}-q\right\|+\lambda_{n} \eta_{n}\left\|x_{n}-q\right\| \\
& \leq\left(1+\lambda_{n}\left(\eta_{n}-1\right)\right)\left\|x_{n}-q\right\| \\
& \leq \eta_{n}\left\|x_{n}-q\right\| .
\end{aligned}
$$

(iii) The definition of $y_{n}$ in (2.1) implies

$$
\begin{aligned}
\left\|y_{n}-q\right\| & =\left\|\left(1-\beta_{n}\right) x_{n}+\beta_{n} T_{2}^{n} z_{n}-q\right\| \\
& \leq\left(1-\beta_{n}\right)\left\|x_{n}-q\right\|+\beta_{n} k_{n}^{2}\left\|z_{n}-q\right\| \\
& \leq\left(1+\beta_{n}\left(k_{n}^{2} \eta_{n}-1\right)\right)\left\|x_{n}-q\right\| \\
& \leq k_{n}^{2} \eta_{n}\left\|x_{n}-q\right\| .
\end{aligned}
$$

By $(i),(i i)$ and $(i i i)$ one can have

$$
\begin{aligned}
\left\|x_{n+1}-q\right\| & =\left\|\left(1-\alpha_{n}\right)\left(x_{n}-q\right)+\alpha_{n}\left(T_{3}^{n} y_{n}-q\right)\right\| \\
& \leq\left(1-\alpha_{n}\right)\left\|x_{n}-q\right\|+\alpha_{n} k_{n}^{3}\left\|y_{n}-q\right\| \\
& \leq\left(1-\alpha_{n}\right)\left\|x_{n}-q\right\|+\alpha_{n} k_{n}^{3} k_{n}^{2} \eta_{n}\left\|x_{n}-q\right\| \\
& \leq\left(1+\alpha_{n}\left(k_{n}^{3} k_{n}^{2} \eta_{n}-1\right)\right)\left\|x_{n}-q\right\| .
\end{aligned}
$$

Since $\sum_{n=1}^{\infty}\left(\eta_{n} k_{n}^{2} k_{n}^{3}-1\right)<\infty$, Lemma 1 and inequality (2.6) imply $\lim _{n \longrightarrow \infty}\left\|x_{n}-q\right\|=r$ exists. Furthermore,

$$
\lim _{n \longrightarrow \infty}\left\|x_{n+1}-q\right\|=\lim _{n \longrightarrow \infty}\left\|\left(1-\alpha_{n}\right)\left(x_{n}-q\right)+\alpha_{n}\left(T_{3}^{n} y_{n}-q\right)\right\|=r .
$$

Also, by (2.5)

$$
\limsup _{n \longrightarrow \infty}\left\|T_{3}^{n} y_{n}-q\right\| \leq \limsup _{n \longrightarrow \infty}\left(k_{n}^{3}\left\|y_{n}-q\right\|\right)
$$




$$
\begin{aligned}
& \leq \limsup _{n \longrightarrow \infty}\left(k_{n}^{3} k_{n}^{2} \eta_{n}\left\|x_{n}-q\right\|\right) \\
& \leq \lim _{n \longrightarrow \infty}\left\|x_{n}-q\right\|=r .
\end{aligned}
$$

Lemma 2 shows that $\lim _{n \rightarrow \infty}\left\|T_{3}^{n} y_{n}-x_{n}\right\|=0$. Furthermore, by (2.6)

$$
\frac{\left\|x_{n+1}-q\right\|-\left\|x_{n}-q\right\|+\alpha_{n}\left\|x_{n}-q\right\|}{\alpha_{n} k_{n}^{3}} \leq\left\|y_{n}-q\right\|,
$$

and by taking the limit $n \longrightarrow \infty$ in (2.7), we get

$$
\lim _{n \longrightarrow \infty}\left\|x_{n}-q\right\| \leq \liminf _{n \longrightarrow \infty}\left\|y_{n}-q\right\| .
$$

By (2.5),

$$
\limsup _{n \longrightarrow \infty}\left\|y_{n}-q\right\| \leq \limsup _{n \longrightarrow \infty}\left(k_{n}^{2} \eta_{n}\left\|x_{n}-q\right\|\right)=\lim _{n \longrightarrow \infty}\left\|x_{n}-q\right\| .
$$

Notice that (2.7) and (2.8) show

$$
\lim _{n \longrightarrow \infty}\left\|x_{n}-q\right\|=\lim _{n \longrightarrow \infty}\left\|y_{n}-q\right\|=r .
$$

Thus

$$
\limsup _{n \longrightarrow \infty}\left\|\left(1-\beta_{n}\right)\left(x_{n}-q\right)+\beta_{n}\left(T_{2}^{n} z_{n}-q\right)\right\|=\limsup _{n \longrightarrow \infty}\left\|y_{n}-q\right\|=r .
$$

On the other hand, (2.4) implies

$$
\begin{aligned}
\limsup _{n \longrightarrow \infty}\left\|T_{2}^{n} z_{n}-q\right\| & \leq \limsup _{n \longrightarrow \infty}\left(k_{n}^{2}\left\|z_{n}-q\right\|\right) \\
& \leq \limsup _{n \longrightarrow \infty}\left(k_{n}^{2} \eta_{n}\left\|x_{n}-q\right\|\right) \\
& \leq \lim _{n \longrightarrow \infty}\left\|x_{n}-q\right\|=r,
\end{aligned}
$$

and Lemma 2 shows that $\lim _{n \rightarrow \infty}\left\|T_{2}^{n} z_{n}-x_{n}\right\|=0$. Using the same technique, we have $\lim _{n \longrightarrow \infty}\left\|z_{n}-q\right\|=r$ and $\lim _{n \rightarrow \infty}\left\|u_{n}-x_{n}\right\|=0$.

Now, we prove $\left\|x_{n}-T_{2}^{n} x_{n}\right\| \longrightarrow 0$. From (2.1),

$$
\left\|z_{n}-x_{n}\right\|=\left\|\left(1-\lambda_{n}\right) x_{n}+\lambda_{n} u_{n}-x_{n}\right\|=\lambda_{n}\left\|u_{n}-x_{n}\right\| \longrightarrow 0, \text { as } n \longrightarrow \infty .
$$

Notice that $\left\|x_{n}-T_{2}^{n} x_{n}\right\| \leq\left\|x_{n}-T_{2}^{n} z_{n}\right\|+\left\|T_{2}^{n} z_{n}-T_{2}^{n} x_{n}\right\|$, where

$$
\begin{aligned}
\left\|T_{2}^{n} z_{n}-T_{2}^{n} x_{n}\right\| \leq & a_{n}^{2}\left\|z_{n}-x_{n}\right\|+b_{n}^{2}\left(\left\|z_{n}-T_{2}^{n} z_{n}\right\|+\left\|T_{2}^{n} x_{n}-x_{n}\right\|\right) \\
& +c_{n}^{2}\left(\left\|T_{2}^{n} z_{n}-x_{n}\right\|+\left\|T_{2}^{n} x_{n}-z_{n}\right\|\right) \\
\leq & a_{n}^{2}\left\|x_{n}-z_{n}\right\|+b_{n}^{2}\left(\left\|z_{n}-x_{n}\right\|+\left\|T_{2}^{n} z_{n}-x_{n}\right\|+\left\|T_{2}^{n} x_{n}-x_{n}\right\|\right) \\
& +c_{n}^{2}\left(\left\|T_{2}^{n} z_{n}-x_{n}\right\|+\left\|x_{n}-z_{n}\right\|+\left\|x_{n}-T_{2}^{n} x_{n}\right\|\right) \\
\leq & \left(a_{n}^{2}+b_{n}^{2}+c_{n}^{2}\right)\left\|x_{n}-z_{n}\right\|+\left(b_{n}^{2}+c_{n}^{2}\right)\left(\left\|T_{2}^{n} z_{n}-x_{n}\right\|+\left\|T_{2}^{n} x_{n}-x_{n}\right\|\right) .
\end{aligned}
$$


Since

$$
\begin{aligned}
\left\|x_{n}-T_{2}^{n} x_{n}\right\| \leq & \left\|x_{n}-T_{2}^{n} z_{n}\right\|+\left(a_{n}^{2}+b_{n}^{2}+c_{n}^{2}\right)\left\|x_{n}-z_{n}\right\| \\
& +\left(b_{n}^{2}+c_{n}^{2}\right)\left(\left\|T_{2}^{n} z_{n}-x_{n}\right\|+\left\|T_{2}^{n} x_{n}-x_{n}\right\|\right)
\end{aligned}
$$

and

$$
\left\|x_{n}-T_{2}^{n} x_{n}\right\| \leq k_{n}^{2}\left\|z_{n}-x_{n}\right\|+\left(1+k_{n}^{2}\right)\left\|x_{n}-T_{2}^{n} z_{n}\right\|,
$$

by inequality (2.10), $\left\|x_{n}-T_{2}^{n} x_{n}\right\| \longrightarrow 0$.

Similarly, $\left\|x_{n}-T_{3}^{n} x_{n}\right\| \longrightarrow 0$. Therefore, by Lemma $6,\left\|x_{n}-T_{i} x_{n}\right\| \longrightarrow 0$ as $n \longrightarrow \infty$, for $i=2,3$.

Since $\lim _{n \rightarrow \infty}\left\|x_{n}-q\right\|$ exists and by the boundedness of $\left\{x_{n}\right\}$, there exists a subsequence $\left\{x_{n k}\right\}$ of $\left\{x_{n}\right\}$ such that $x_{n k} \rightarrow x$. Lemma 5 shows $x \in F$. Notice $w_{w}\left(\left\{x_{n}\right\}\right)$ is a singleton. To do this, suppose there exists another subsequence $\left\{x_{n j}\right\}$ of $\left\{x_{n}\right\}$ which is weakly convergent to some $z \neq x$ such that $z \in F$. The existence of $\lim _{n \rightarrow \infty}\left\|x_{n}-q\right\|$ implies the existence of $\lim _{n \rightarrow \infty}\left\|x_{n}-x\right\|$ and $\lim _{n \rightarrow \infty}\left\|x_{n}-z\right\|$. Since $E$ satisfies the Opial's condition, we have

$$
\lim _{n \rightarrow \infty}\left\|x_{n}-x\right\|=\lim _{k \rightarrow \infty}\left\|x_{n k}-x\right\|<\lim _{k \rightarrow \infty}\left\|x_{n k}-z\right\|=\lim _{n \rightarrow \infty}\left\|x_{n}-z\right\|,
$$

and

$$
\lim _{n \rightarrow \infty}\left\|x_{n}-z\right\|=\lim _{j \rightarrow \infty}\left\|x_{n j}-z\right\|<\lim _{j \rightarrow \infty}\left\|x_{n j}-x\right\|=\lim _{n \rightarrow \infty}\left\|x_{n}-x\right\|,
$$

which leads to a contradiction, and $w_{w}\left(\left\{x_{n}\right\}\right)$ is a singleton. Therefore by Lemma 3 $\left\{x_{n}\right\}$ is weakly convergent to $x$.

With respect to Theorem 2, the following example is presented.

Example 2. Let $E=\mathbb{R}, C=[0, \infty)$. Assume $\left\{x_{n}\right\}$ is the sequence defined by (2.1), where $T_{1} x=\frac{x}{100}, T_{2} x=\frac{x}{1000}$ and $T_{3} x=\frac{x}{10000}$. Also $\alpha_{n}=\frac{n}{3 n+1}, \beta_{n}=\frac{n}{4 n+1}$ and $\lambda_{n}=\frac{n}{5 n+1}$. We have

$$
\left\{\begin{array}{l}
u_{n}=\frac{1}{n+1} \sum_{j=0}^{n} \frac{x_{n}}{100^{j}}, \\
z_{n}=\frac{4 n+1}{5 n+1} x_{n}+\left(\frac{n}{5 n+1}\right) u_{n}, \\
y_{n}=\frac{3 n+1}{4 n+1} x_{n}+\left(\frac{n}{4 n+1}\right)\left(\frac{1}{1000^{n}}\right) z_{n}, \\
x_{n+1}=\frac{2 n+1}{3 n+1} x_{n}+\left(\frac{n}{3 n+1}\right)\left(\frac{1}{10000^{n}}\right) y_{n} .
\end{array}\right.
$$

Let $x_{1}=1$, then by iteration we can have

$$
x_{10}=0.03842, \cdots, x_{20}=0.00074, \cdots, x_{30}=0.00001, \cdots
$$

This shows $x_{n} \rightarrow 0$. Thus 0 is a common fixed point of $T_{1}, T_{2}$ and $T_{3}$ or $\{0\}=\bigcap_{i=1}^{3} F\left(T_{i}\right)$. 


\section{REFERENCES}

[1] R. P. Agarwal, D. O'Regan, and D. R. Sahu, "Iterative construction of fixed points of nearly asymptotically nonexpansive mappings." J. Nonlinear Convex Anal., vol. 8, pp. 61-79, 2007.

[2] N. Akkasriworn, A. Kaewkhao, A. Keawkhao, and K. Sokhuma, "Common fixed-point results in uniformly convex Banach spaces." Fixed Point Theory and Applications, vol. 2012, no. 171, pp. 1-7, 2012, doi: 10.1186/1687-1812-2012-171.

[3] J. B. Baillon, "Un thèoreme de type ergodique pour les contractions non linèaires dans un espace de Hilbert." C.R. Acad. Sci. Paris Ser. A-B., vol. 280, pp. 1511-1541, 1975.

[4] V. Berinde, "Asymptotic Regularity, Fixed Points and Successive Approximations." Filomat, vol. 34, no. 3, pp. 965-981, 2020.

[5] Y. J. Cho, J. I. Kang, and H. Zrou, "Approximating common fixed points of asymptotically nonexpansive mappings." Bull. Korean Math. Soc., vol. 42, pp. 661-670, 2005, doi: 10.4134/BKMS.2005.42.4.661.

[6] J. A. Clarkson, "Uniformly convex spaces." Trans. Am. Math. Soc., vol. 40, pp. 396-414, 1936.

[7] M. B. Ghaemi and A. Razani, "Fixed and periodic points in the probabilistic normed and metric spaces." Chaos, Solitons and Fractals, vol. 28, pp. 1181-1187, 2006, doi: 10.1016/j.chaos.2005.08.192.

[8] J. S. Jung and B. S. Thakur, "Fixed point theorems for generalized Lipschitzian semigroups." Int. J. Math. Math. Sci., vol. 28, pp. 41-50, 2001.

[9] M. A. Ragusa, "Regularity of solutions of divergence form elliptic equations." Proceedings of the American Mathematical Society, vol. 128, no. 2, pp. 533-540, 2000, doi: 10.1090/S0002-993999-05165-5.

[10] M. A. Ragusa and A. Tachikawa, "Partial regularity of the minimizers of quadratic functionals with VMO coefficients." Journal of the London Mathematical Society-second series, vol. 72, no. 3, pp. 609-620, 2005, doi: 10.1112/S002461070500699X.

[11] A. Razani, "A fixed point theorem in the Menger probabilistic metric space." New Zealand J. Math., vol. 35, no. 1, pp. 109-114, 2006.

[12] A. Razani, "Existence of fixed point for the nonexpansive mapping of intuitionistic fuzzy metric spaces." Chaos, Solitons and Fractals, vol. 30, no. 2, pp. 367-373, 2006, doi: 10.1016/j.chaos.2005.10.010.

[13] A. Razani, Results in Fixed Point Theory. Q Qazvin: Andisheh Zarin publisher, 2010.

[14] A. Razani, "An existence theorem for ordinary differential equation in Menger probabilistic metric space." Miskolc Mathematical Notes, vol. 15, no. 2, pp. 711-716, 2014, doi: 10.18514/MMN.2014.640.

[15] A. Razani and R. Moradi, Fixed Point Theory in Modular Space. Qazvin: Saieh Ghostar publisher, 2006

[16] H. R. Sahebi and A. Razani, "A solution of a general equilibrium problem." Acta Mathematica Scientia, vol. 33, no. 6, pp. 1598-1614, 2013.

[17] J. Schu, "Iterative construction of fixed points of asymptotically nonexpansive mappings." J. Math. Anal. Appl., vol. 158, no. 2, pp. 407-413, 1991, doi: 10.1016/0022-247X(91)90245-U.

[18] T. Shimizu and W. Takahashi, "Strong convergence to common fixed points of nonexpansive mappings." J. Math. Anal. Appl., vol. 211, pp. 71-83, 1997, doi: 10.1006/jmaa.1997.5398.

[19] T. Shioji and W. Takahashi, "A strong convergence theorem for asymptotically nonexpansive mappings in Banach spaces." Arch. Math., vol. 72, pp. 354-359, 1999, doi: $10.1007 / \mathrm{s} 000130050343$.

[20] W. Takahashi, Nonlinear Functional Analysis: fixed point theory and its applications. New York: Yokohama Publishers, 2000. 
[21] K. K. Tan and H. K. Xu, "The nonlinear ergodic theorem for asymptotically nonexpansive mappings in Banach spaces." Proc. Amer. Math. Soc., vol. 114, no. 2, pp. 399-404, 1992, doi: 10.1090/S0002-9939-1992-1068133-2.

[22] K. K. Tan and H. K. Xu, "Fixed point iterative processes for asymptotically nonexpansive mappings." Proc. Amer. Math. Soc., vol. 122, no. 3, pp. 733-739, 1994, doi: 10.1090/S0002-99391994-1203993-5.

[23] H. K. Xu, "Inequalities in Banach spaces with applications." Nonlinear Anal. TMA, vol. 16, no. 12, pp. 1127-1138, 1991, doi: /10.1016/0362-546X(91)90200-K.

Author's address

A. Razani

Imam Khomeini International University, Faculty of Science, Department of Pure Mathematics, Postal Code: 34149-16818 Qazvin, IRAN

E-mail address: razaniesci.ikiu.ac.ir 